\section{(2) \\ BRAZIULIAN JOURNAL \\ OF MEDICAL AND BIOLOGICAL RESEARCH}

www.bjournal.com.br
ISSN 1414-431X

Volume 45 (11) 995-1101 November 2012

BIOMEDICAL SCIENCES

AND

CLINICAL INVESTIGATION

Braz J Med Biol Res, November 2012, Volume 45(11) 1095-1101

doi: $10.1590 / \mathrm{S} 0100-879 \mathrm{X} 2012007500118$

Differences in synthesis and absorption of cholesterol of two effective lipid-lowering therapies

S.H. Kasmas, M.C. Izar, C.N. França, S.C. Ramos, F.T. Moreira, T. Helfenstein, R.A. Moreno, N.C. Borges, A.M. Figueiredo-Neto and F.A. Fonseca

The Brazilian Journal of Medical and Biological Research is partially financed by

\section{Q}

Ministério da Ciência e Tecnologia

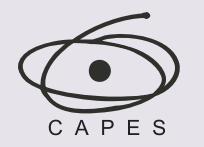

Ministério da Educação
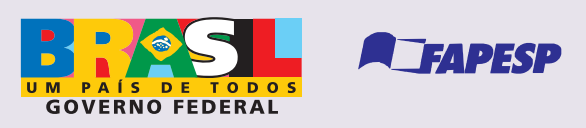

Institutional Sponsors

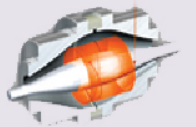

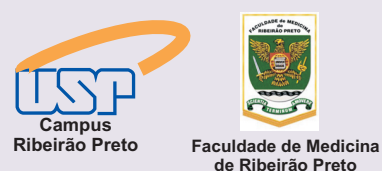

de Ribeirão Preto

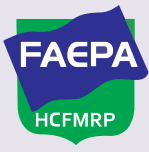

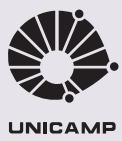

SHIMADZU

UNICAMP

)

Associaçaco

Associaçăo
Fundo de Incentivo
à Pesquisa

\section{analitica Thermo}




\title{
Differences in synthesis and absorption of cholesterol of two effective lipid-lowering therapies
}

\author{
S.H. Kasmas ${ }^{1}$, M.C. Izar ${ }^{1,2}$, C.N. França ${ }^{1}$, S.C. Ramos ${ }^{1}$, F.T. Moreira ${ }^{1}$, T. Helfenstein ${ }^{1,2}$, \\ R.A. Moreno ${ }^{3}$, N.C. Borges ${ }^{3}$, A.M. Figueiredo-Neto ${ }^{2}$ and F.A. Fonseca1,2 \\ ${ }^{1}$ Divisão de Cardiologia, Departamento de Medicina, Universidade Federal de São Paulo, São Paulo, SP, Brasil \\ 2Instituto Nacional de Ciência e Tecnologia de Fluidos Complexos, São Paulo, SP, Brasil \\ ${ }^{3}$ Synchrophar, Campinas, SP, Brasil
}

\begin{abstract}
Effective statin therapy is associated with a marked reduction of cardiovascular events. However, the explanation for full benefits obtained for LDL cholesterol targets by combined lipid-lowering therapy is controversial. Our study compared the effects of two equally effective lipid-lowering strategies on markers of cholesterol synthesis and absorption. A prospective, open label, randomized, parallel design study, with blinded endpoints, included 116 subjects. We compared the effects of a 12-week treatment with $40 \mathrm{mg}$ rosuvastatin or the combination of $40 \mathrm{mg}$ simvastatin/10 mg ezetimibe on markers of cholesterol absorption (campesterol and $\beta$-sitosterol), synthesis (desmosterol), and their ratios to cholesterol. Both therapies similarly decreased total and LDL cholesterol, triglycerides and apolipoprotein B, and increased apolipoprotein A1 $(P<0.05$ vs baseline for all). Simvastatin/ezetimibe increased plasma desmosterol $(P=0.012$ vs baseline), and decreased campesterol and $\beta$-sitosterol $(P<0.0001$ vs baseline for both), with higher desmosterol $(P=0.007)$ and lower campesterol and $\beta$-sitosterol compared to rosuvastatin, $(P<0.0001$, for both). In addition, rosuvastatin increased the ratios of these markers to cholesterol $(P<0.002$ vs baseline for all), whereas simvastatin/ezetimibe significantly decreased the campesterol/cholesterol ratio $(P=0.008$ vs baseline $)$ and tripled the desmosterol/cholesterol ratio $(P<0.0001 \mathrm{vs}$ baseline). The campesterol/cholesterol and $\beta$-sitosterol/cholesterol ratios were lower, whereas the desmosterol/cholesterol ratio was higher in patients receiving simvastatin/ezetimibe $(P<0.0001$ vs rosuvastatin, for all). Pronounced differences in markers of cholesterol absorption and synthesis were observed between two equally effective lipid-lowering strategies.
\end{abstract}

Key words: Campesterol; $\beta$-sitosterol; Desmosterol; Statin; Ezetimibe

\section{Introduction}

A recent meta-analysis has shown that marked reductions in LDL cholesterol with statins are related to a greater absolute decrease in major cardiovascular events (1). The most appropriate strategies for achieving greater effectiveness in cholesterol reduction include the use of high doses of potent statins or the combination of lipid-lowering drugs $(2,3)$. However, depending on the choice of drugs, significant differences in cholesterol absorption and synthesis can be expected $(4,5)$.

Indeed, reduction in the endogenous synthesis of cholesterol by statins can be accompanied by increased intestinal sterol absorption (6). On the other hand, ezetimibe, an inhibitor of dietary and biliary intestinal cholesterol absorption, has been extensively used as a lipid-lowering agent, but its role in endogenous cholesterol synthesis is less studied, especially when combined with a statin (7).

Differences in the pharmacokinetic properties of statins may account for the effectiveness of cholesterol synthesis inhibition as well as for the stimuli of cholesterol absorption. Ezetimibe has an estimated half-life of approximately 22 h (8), while simvastatin was reported to have a relatively short half-life of less than $3 \mathrm{~h}(9)$.

Recently, the phenotype of high absorption and low synthesis of cholesterol has been related to increased cardiovascular and all-cause mortality (10). On the other hand, some additional benefits of statins, including antiinflammatory and antithrombotic properties, or improvement of endothelial function are related to the lower expression

Correspondence: F.A. Fonseca, Divisão de Cardiologia, Departamento de Medicina, UNIFESP, Rua Pedro de Toledo, 276, 04039-030 São Paulo, SP, Brasil. Fax: +55-11-5084-8777. E-mail: fahfonseca@terra.com.br or ffonseca@cardiol.br

Received February 17, 2012. Accepted May 10, 2012. Available online July 20, 2012. Published October 5, 2012. 
of intermediate compounds of the endogenous cholesterol synthesis pathway (11-13).

Therefore, the overall benefit from lipid-lowering therapy for cardiovascular disease prevention seems to depend on the effective reduction in both endogenous cholesterol synthesis and intestinal absorption of sterols. Since simvastatin is a statin with a short half-life and lower affinity for hydroxymethylglutaryl-coenzyme A reductase than the latest generation of statins $(14,15)$, we hypothesized that this statin could be insufficient to counterbalance the stimulus of cholesterol synthesis promoted by ezetimibe.

Thus, we compared the effects of two potent and equally effective lipid-lowering strategies on markers of cholesterol synthesis and absorption.

\section{Material and Methods}

\section{Design and study population}

We performed a prospective, randomized, open-label study, with parallel arms and blinded endpoints. Patients were recruited from the outpatient dyslipidemia unit of the Universidade Federal de São Paulo. The trial protocol was conducted in accordance with the ethical standards of the Universidade Federal de São Paulo on human experimentation and approval was obtained from the Universidade Federal de São Paulo Ethics Committee. Written informed consent was obtained from all subjects prior to inclusion.

Eligible patients were men and women, 30 to 75 years of age, under treatment for primary or secondary prevention of coronary heart disease, who had an indication for lipid-lowering therapy according to the National Cholesterol Education Program/Adult Treatment Panel III (NCEP/ATP) guidelines (16). A total of 116 subjects completed the study protocol. Patients with liver, renal or gastrointestinal disease, malignancies, and uncontrolled metabolic disorders that might affect the tolerability or safety of the treatments were excluded. Exclusion criteria during the study were low adherence (less than $80 \%$ ) to the lipid-lowering regimen. The major characteristics of the study population are listed in Table 1. Risk factors were defined by the NCEP/ATP III guidelines (16). Before treatment, all patients received nutrition counseling based on the Therapeutic Lifestyle Changes of the NCEPIATP III (16). They were then randomized to receive $40 \mathrm{mg}$ rosuvastatin or the combination of $40 \mathrm{mg}$ simvastatin plus $10 \mathrm{mg}$ ezetimibe, daily for 12 weeks. During the study, patients were evaluated at 4-week intervals, when compliance with study medication was confirmed. The patients did not take lipid-lowering agents 4 weeks before the beginning of the study.

\section{Study drugs}

Rosuvastatin (Crestor ${ }^{\circledR}$, IPR Pharmaceuticals, Porto Rico) and simvastatin/ezetimibe (Zetsim ${ }^{\circledR}$, Schering-Plough Products, Porto Rico) were supplied by AstraZeneca (Brazil) and Merck Co. (Brazil), respectively.

\section{Blood sample collection and assays}

Biochemical analyses were performed in samples obtained after a 12-h fasting period at baseline and after 12 weeks of treatment and were processed by a central laboratory of the Universidade Federal de São Paulo using automated techniques.

\section{Lipids and biochemistry}

Serum total cholesterol, HDL cholesterol, and triglycerides were determined by automated methods (Advia 2400, Siemens Healthcare Diagnostics, Japan). LDL cholesterol was calculated using the Friedewald equation (17). Apolipoprotein $A 1$, apolipoprotein $B$, and highly sensitive C-reactive protein (hs-CRP) were determined by nephelometry (Array 360 CE/AL, Beckmann Coulter, Inc., USA). Alanine aminotransferase (ALT), aspartate aminotransferase (AST), creatinine, and creatine kinase (CK) were assayed by automated techniques (Advia 2400, Siemens Healthcare Diagnostics).

\section{Markers of sterol absorption and synthesis}

For the quantification of $\beta$-sitosterol and campesterol (markers of sterol absorption), as well as for desmosterol (a precursor of endogenous cholesterol synthesis), we used ultra-performance liquid chromatography (UPLC) and mass spectrometry (MS) as previously reported $(18,19)$. Briefly, the method consisted of liquid-liquid extraction followed by separation in the UPLC system and detection with an atmospheric pressure chemical ionization (APCI) ion source mass spectrometer operating on "single ion monitoring" for each sterol ( $\beta$-sitosterol, campesterol, and desmosterol). The MS system (Quattro Premier-XE, Waters Co., UK) was adjusted to monitor single ions formed by an APCI ion source. The sterols were detected as their free forms, i.e., non-esterified, with the ions being monitored with a mass to charge ratio $(\mathrm{m} / \mathrm{z})$ of 367.30 for desmosterol, 397.25 for $\beta$-sitosterol, and 383.60 for campesterol. Absolute values of plasma sterol levels were reported as $\mathrm{mg} / \mathrm{dL}$. As these sterols are transported by lipoproteins, absolute values of sterols were corrected for total plasma cholesterol and reported as their ratios to cholesterol.

\section{Statistical analyses}

Numerical data are reported as means \pm SEM or medians (interquartile range). Categorical data are reported as number (\%) and compared by the Pearson chi-square test. Continuous variables were tested for distribution of normality by the Kolmogorov-Smirnov test. Variables with Gaussian distribution were compared between times and groups using a general linear model (GLM) - repeated measures. When data were not normally distributed, we used Mann-Whitney or Wilcoxon tests for comparisons between groups and within groups, respectively. Statistical significance was set at $P<0.05$. All analyses were two-tailed 
and were carried out using the SPSS 17.0 for Windows (SPSS Inc., USA).

\section{Results}

\section{Patients}

A total of 116 subjects presented $>80 \%$ adherence to the study protocol. The major characteristics of these subjects were comparable between groups at baseline (Table 1).

\section{Lipids, apolipoproteins, and other laboratory parameters}

At baseline, biochemical analyses including lipid profile and apolipoproteins did not differ between groups (Table 2). A 12-week treatment with either $40 \mathrm{mg}$ rosuvastatin or $40 \mathrm{mg}$ simvastatin/10 mg ezetimibe promoted similar reductions of total and LDL cholesterol, as well as of serum triglyceride levels, without changes in HDL cholesterol. Both regimens increased serum apolipoprotein $A 1$ and reduced serum apolipoprotein $B$ levels without differences between treatment arms (Table 2).

Creatinine and glucose did not differ between groups along the study. AST, ALT and CK were slightly increased after the treatments, but remained within the normal range. hs-CRP levels decreased similarly in each treatment group (Table 3).

\section{Campesterol, $\beta$-sitosterol and desmosterol}

At baseline, plasma levels of campesterol, $\beta$-sitosterol and desmosterol were comparable between groups. Treatment with high-dose rosuvastatin $(40 \mathrm{mg})$ did not change the plasma markers of sterol absorption and endogenous cholesterol synthesis over a period of 12 weeks $(P=N S$, Wilcoxon test) (Table 4, Figure 1A-C). Conversely, in subjects receiving $40 \mathrm{mg}$ simvastatin/10 mg ezetimibe, we observed increased cholesterol synthesis, as shown by the increase in plasma desmosterol levels $(P=0.012)$ and by the decrease in cholesterol absorption markers ( $P$ $<0.0001$ for both campesterol and $\beta$-sitosterol) at the end of treatment compared to their baseline levels (Table 4, Figure $1 \mathrm{~A}-\mathrm{C})$. In addition, patients treated with the combination of simvastatin/ezetimibe presented lower plasma campesterol and $\beta$-sitosterol levels than those receiving rosuvastatin $(P<0.0001)$; these patients also presented higher plasma desmosterol levels $(P=0.007)$ compared to rosuvastatin-treated subjects.

The ratios between absorption markers and cholesterol (campesterol/cholesterol, $\beta$-sitosterol/cholesterol) and between synthesis markers and cholesterol (desmosterol/ cholesterol) were also comparable between groups at baseline $(P=N S)$. Since rosuvastatin reduced total and LDL cholesterol levels, the synthesis and absorption ratios for cholesterol were all increased over time $(P<0.002$ vs baseline). Treatment with simvastatin/ezetimibe decreased the campesterol/cholesterol ratio $(P=0.008$ vs baseline $)$, but
Table 1. Baseline characteristics of the study population according to treatment assignment.

\begin{tabular}{lcc}
\hline Characteristic & $\begin{array}{c}\text { Rosuvastatin } \\
(\mathrm{N}=58)\end{array}$ & $\begin{array}{c}\text { Simvastatin/ezetimibe } \\
(\mathrm{N}=58)\end{array}$ \\
\hline Age, years, median (IQ) & $59(53-65)$ & $59(54-65)$ \\
Male gender, N (\%) & $17(29.3)$ & $18(31.0)$ \\
Diabetes mellitus, N (\%) & $12(20.7)$ & $14(24.1)$ \\
Hypertension, N (\%) & $46(79.3)$ & $44(75.9)$ \\
Smoking, N (\%) & $5(8.6)$ & $4(6.9)$ \\
Metabolic syndrome, N (\%) & $35(60.3)$ & $27(46.6)$ \\
Primary prevention, N (\%) & $47(81.0)$ & $48(82.8)$ \\
\hline
\end{tabular}

There were no statistical differences between groups (chi-square test). IQ = interquartile range. Metabolic syndrome according to the National Cholesterol Education Program/Adult Treatment Program III (NCEP/ATP III) guidelines (16).

Table 2. Lipid and apolipoprotein parameters of the two groups at baseline and at the end of the study.

\begin{tabular}{|c|c|c|c|}
\hline Parameter & $\begin{array}{l}\text { Rosuvastatin } \\
\quad(N=58)\end{array}$ & $\begin{array}{l}\text { Simvastatin/ezetimibe } \\
\qquad(N=58)\end{array}$ & $P(w g)$ \\
\hline \multicolumn{4}{|l|}{ Cholesterol } \\
\hline Baseline & $250 \pm 6$ & $243 \pm 6$ & $<0.0001$ \\
\hline End of study & $144 \pm 4$ & $150 \pm 6$ & \\
\hline \multicolumn{4}{|l|}{ LDL-C } \\
\hline Baseline & $161 \pm 5$ & $160 \pm 5$ & $<0.0001$ \\
\hline End of study & $67 \pm 3$ & $76 \pm 4$ & \\
\hline \multicolumn{4}{|l|}{ HDL-C } \\
\hline Baseline & $54 \pm 2$ & $52 \pm 2$ & 0.38 \\
\hline End of study & $53 \pm 2$ & $52 \pm 2$ & \\
\hline \multicolumn{4}{|l|}{ Triglycerides } \\
\hline Baseline & $173 \pm 11$ & $152 \pm 9$ & $<0.0001$ \\
\hline End of study & $117 \pm 6$ & $110 \pm 6$ & \\
\hline \multicolumn{4}{|l|}{ Apo A1 } \\
\hline Baseline & $150 \pm 4$ & $142 \pm 3$ & 0.02 \\
\hline End of study & $152 \pm 4$ & $148 \pm 3$ & \\
\hline \multicolumn{4}{|l|}{ Apo B } \\
\hline Baseline & $131 \pm 4$ & $133 \pm 4$ & $<0.0001$ \\
\hline End of study & $67 \pm 2$ & $71 \pm 3$ & \\
\hline
\end{tabular}

Lipids and apolipoproteins are reported as means \pm SEM in $\mathrm{mg} /$ dL. LDL-C and HDL-C = low- and high-density lipoprotein cholesterol, respectively; Apo = apolipoprotein. There were significant statistical differences within groups (wg) (general linear model with repeated measures test); however, there were no significant differences between groups (general linear model with repeated measures test).

did not change the $\beta$-sitosterol/cholesterol ratio $(P=0.960$ vs baseline), and increased the desmosterol/cholesterol ratio $(P<0.0001$ vs baseline). In addition, the campesterol/ cholesterol and $\beta$-sitosterol/cholesterol ratios were lower 
and the desmosterol/cholesterol ratio was higher in patients receiving simvastatin/ezetimibe than in those treated with rosuvastatin $(P<0.0001$, for all; Table 4, Figure 2A-C).

\section{Discussion}

We found important differences between the two lipid-lowering regimens in the balance of markers of sterol absorption and synthesis, in spite of a similar decrease in serum LDL cholesterol levels. Combined therapy with simvastatin plus ezetimibe reduced the markers of cholesterol absorption and promoted an increase in plasma desmosterol levels, suggesting that the inhibition of endogenous cholesterol synthesis by simvastatin is not sufficient to avert the increased endogenous synthesis following the blockade of cholesterol absorption by ezetimibe. Conversely, treatment with rosuvastatin resulted in a similar decrease in cholesterol levels without changes in absolute campesterol, $\beta$-sitosterol or desmosterol values. However, due to the higher degree of cholesterol synthesis inhibition observed with potent statins alone, the ratios between markers of absorption and synthesis and cholesterol differed between treatment groups, showing exacerbation of the effect of ezetimibe increasing endogenous cholesterol synthesis and attenuating the expected inhibition of cholesterol absorption when used in combination with simvastatin. The reduction in LDL cholesterol observed with lipid-lowering agents per se is a stimulus for the increase in cholesterol synthesis, regardless of the mechanism by which cholesterol is reduced. Therefore, when combining a statin with a short half-life with an inhibitor of cholesterol absorption with a long half-life, we can expect a more pronounced increase in cholesterol synthesis markers. Conversely, if a potent statin with a long half-life is used alone, a more prominent inhibition of cholesterol synthesis can be expected.

Interestingly, the effects on intestinal absorption markers may differ among statins. The maximal doses of atorvastatin and rosuvastatin were compared in a study in which the authors found a modest absolute decrease in campesterol and sitosterol levels with rosuvastatin, and an increase in these cholesterol absorption markers with atorvastatin (5). The authors postulated an interaction between ABCG5/G8 transporters and atorvastatin to explain their findings (20). Other factors, such as the presence of diabetes, have been reported to modify the magnitude of cholesterol synthesis inhibition following lipid-lowering therapies (21).

The relationship between phytosterols and the severity of coronary disease was described in the Ludwigshafen Risk and Cardiovascular Health (LURIC) study (22), which reported data for 2440 individuals in whom coronary angio-
Table 3. Laboratory parameters by groups at baseline and at the end of the study.

\begin{tabular}{|c|c|c|c|}
\hline Parameter & $\begin{array}{l}\text { Rosuvastatin } \\
\qquad(\mathrm{N}=58)\end{array}$ & $\begin{array}{l}\text { Simvastatin/ezetimibe } \\
\qquad(N=58)\end{array}$ & $P(w g)$ \\
\hline \multicolumn{4}{|c|}{ Creatinine (mg/dL) } \\
\hline Baseline & $0.93 \pm 0.03$ & $0.92 \pm 0.03$ & 0.99 \\
\hline End of study & $0.91 \pm 0.03$ & $0.94 \pm 0.03$ & \\
\hline \multicolumn{4}{|l|}{ AST (IU/L) } \\
\hline Baseline & 22 (19-25) & $22(19-25)$ & $0.001^{R}$ \\
\hline End of study & $25(20-32)$ & $24(21-29)$ & \\
\hline \multicolumn{4}{|l|}{ ALT (IU/L) } \\
\hline Baseline & $20(16-25)$ & $21(17-30)$ & $0.001^{\mathrm{R}}$ \\
\hline End of study & $23(17-37)$ & $27(19-32)$ & $<0.0001^{S / E}$ \\
\hline \multicolumn{4}{|l|}{ CK (IU/L) } \\
\hline Baseline & $104(84-159)$ & $127(81-167)$ & $<0.0001^{\mathrm{R}}$ \\
\hline End of study & 135 (106-204) & $118(95-193)$ & $0.02^{S / E}$ \\
\hline \multicolumn{4}{|l|}{ hs-CRP (mg/L) } \\
\hline Baseline & $2.7(1.2-5.4)$ & $2.7(1.6-5.4)$ & $<0.0001^{R}$ \\
\hline End of study & $1.5(0.9-3.5)$ & $1.6(0.7-3.2)$ & $<0.0001^{S / E}$ \\
\hline \multicolumn{4}{|l|}{ Glucose } \\
\hline Baseline & $97(88-110)$ & $97(86-107)$ & $0.67^{R}$ \\
\hline End of study & $97(90-105)$ & $97(89-110)$ & $0.09 S / E$ \\
\hline
\end{tabular}

$\mathrm{AST}=$ aspartate aminotransferase; $\mathrm{ALT}=$ alanine aminotransferase; $\mathrm{CK}=$ creatine kinase; hs-CRP = highly sensitive C-reactive protein. Creatinine is reported as means \pm SEM and was analyzed by the general linear model with repeated measures. AST, ALT, CK, hs-CRP, and glucose are reported as median and interquartile range and were not normally distributed. They were analyzed by the Wilcoxon test (for within-group comparisons) or the Mann-Whitney test (for between-group comparisons). There were significant statistical differences within groups (wg); however, there were no significant differences between groups. ${ }^{R}$ Comparisons for rosuvastatin between visits; ${ }^{S / E_{C}}$ comparisons for simvastatin/ezetimibe between visits.

grams and sterol levels were evaluated. The phenotype of low cholesterol synthesis combined with high cholesterol absorption showed an association with severity of coronary disease. In agreement with these findings, the Framingham Offspring Study also showed that the low-synthesis and high-absorption phenotypes were independent predictors of cardiovascular disease (23). In addition, these markers of absorption and synthesis are also related to HDL cholesterol concentrations (24).

Phytosterolemia is a rare genetic disorder characterized by a substantial increase in plasma levels of plant sterols and premature atherosclerosis $(25,26)$. However, the role of modest increases in plasma phytosterol levels in the risk for coronary disease is controversial (22,27-30).

Reduction of sterol absorption can prevent the increase in cholesterol and plasma plant sterol levels. However, the results of our study suggest the need for an effective statin to avoid the increase in endogenous cholesterol synthesis by the inhibition of cholesterol absorption.

In our study, all patients received nutrition counseling 
Table 4. Markers of cholesterol absorption and synthesis and their ratios to cholesterol by groups at baseline and at the end of the study.

\begin{tabular}{|c|c|c|c|c|}
\hline Parameter & Rosuvastatin & Simvastatin/ezetimibe & $P(b g)$ & $P(w g)$ \\
\hline \multicolumn{5}{|l|}{ Campesterol (mg/dL) } \\
\hline \multicolumn{5}{|l|}{ Baseline } \\
\hline Absolute & $0.97(0.57-1.48)$ & $0.89(0.53-1.51)$ & 0.732 & $0.263^{R}$ \\
\hline Ratio to cholesterol & $0.0037(0.0024-0.0060)$ & $0.0035(0.0024-0.0070)$ & 0.808 & $<0.0001^{R}$ \\
\hline \multicolumn{5}{|l|}{ End of study } \\
\hline Absolute & $0.96(0.76-1.48)$ & $0.40(0.29-0.57)$ & $<0.0001$ & $<0.0001^{S / E}$ \\
\hline Ratio to cholesterol & $0.0072(0.0053-0.0093)$ & $0.0028(0.0019-0.0040)$ & $<0.0001$ & $0.008^{S / E}$ \\
\hline \multicolumn{5}{|l|}{ ß-sitosterol (mg/dL) } \\
\hline \multicolumn{5}{|l|}{ Baseline } \\
\hline Absolute & $0.66(0.42-1.00)$ & $0.61(0.40-1.06)$ & 0.910 & $0.382^{\mathrm{R}}$ \\
\hline Ratio to cholesterol & $0.0027(0.0018-0.0039)$ & $0.0029(0.0016-0.0043)$ & 0.791 & $<0.0001^{R}$ \\
\hline \multicolumn{5}{|l|}{ End of study } \\
\hline Absolute & $0.72(0.51-0.99)$ & $0.40(0.26-0.58)$ & $<0.0001$ & $<0.0001^{S / E}$ \\
\hline Ratio to cholesterol & $0.0050(0.0036-0.0066)$ & $0.0030(0.0018-0.0040)$ & $<0.0001$ & $0.960^{S / E}$ \\
\hline \multicolumn{5}{|l|}{ Desmosterol (mg/dL) } \\
\hline \multicolumn{5}{|l|}{ Baseline } \\
\hline Absolute & $0.39(0.15-0.85)$ & $0.33(0.14-1.02)$ & 0.987 & $0.972^{R}$ \\
\hline Ratio to cholesterol & $0.0014(0.0006-0.0031)$ & $0.0014(0.0005-0.0034)$ & 0.886 & $0.001^{R}$ \\
\hline \multicolumn{5}{|l|}{ End of study } \\
\hline Absolute & $0.43(0.22-0.72)$ & $0.62(0.45-1.02)$ & 0.007 & $0.012^{S / E}$ \\
\hline Ratio to cholesterol & $0.0033(0.0017-0.0051)$ & $0.0046(0.0027-0.0071)$ & 0.010 & $<0.0001^{S / E}$ \\
\hline
\end{tabular}

Data as reported as medians (25th-75th percentiles). ${ }^{R}$ Comparisons for rosuvastatin between visits;

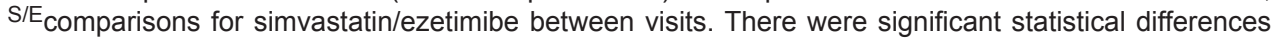
both between (bg; Mann-Whitney test) and within groups (wg; Wilcoxon test).

according to NCEP III guidelines (16). A novel mechanism for the reduction of intestinal sterol absorption following the intake of plant sterols has been recently described. Interestingly, despite the competition between cholesterol and
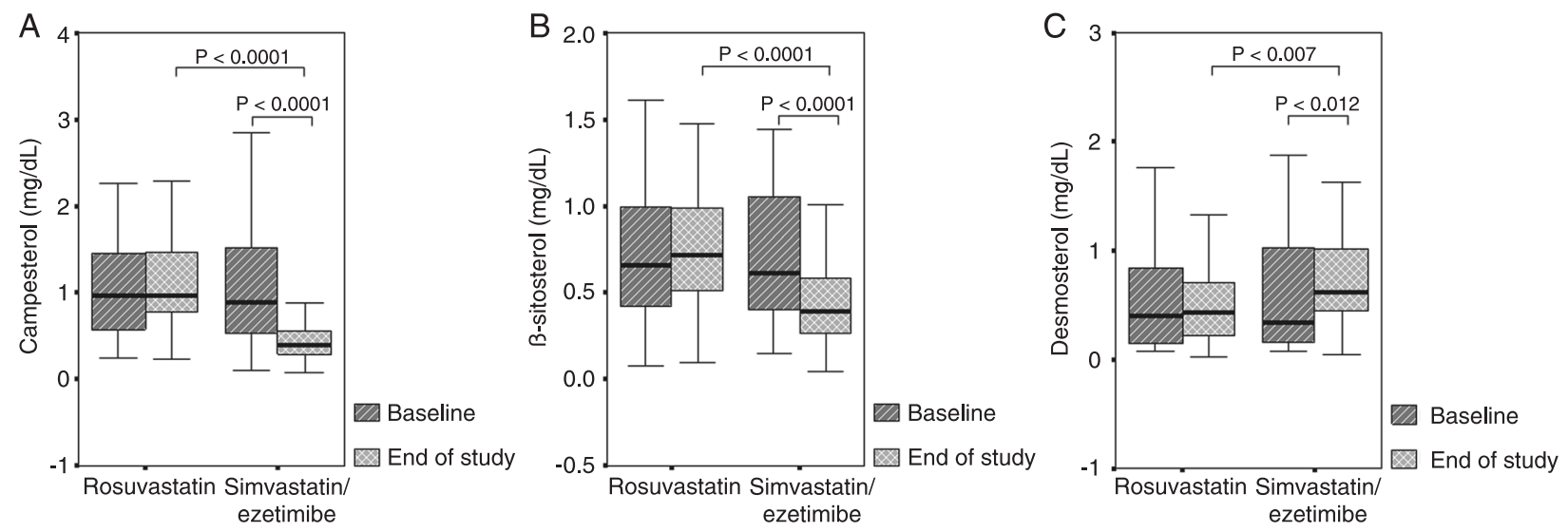

Figure 1. Box-plots (median, 25th and 75th percentiles) for plasma levels of campesterol $(A), \beta$-sitosterol $(B)$, and desmosterol $(C)$ at baseline and at the end of the study according to treatment. Baseline values were comparable between groups. Rosuvastatin did not modify these parameters over time. Subjects treated with simvastatin/ezetimibe presented a reduction of plasma levels of campesterol and $\beta$-sitosterol $(P$ $<0.0001$ vs baseline for both, Wilcoxon test), and an increase of desmosterol ( $P=0.012$ vs baseline, Wilcoxon test). Plasma campesterol and $\beta$-sitosterol levels were lower at the end of the study $(P<0.0001$ for both, Mann-Whitney test) in subjects receiving simvastatin/ezetimibe compared to individuals receiving rosuvastatin, whereas desmosterol levels were higher ( $P=0.007$, Mann-Whitney test). 

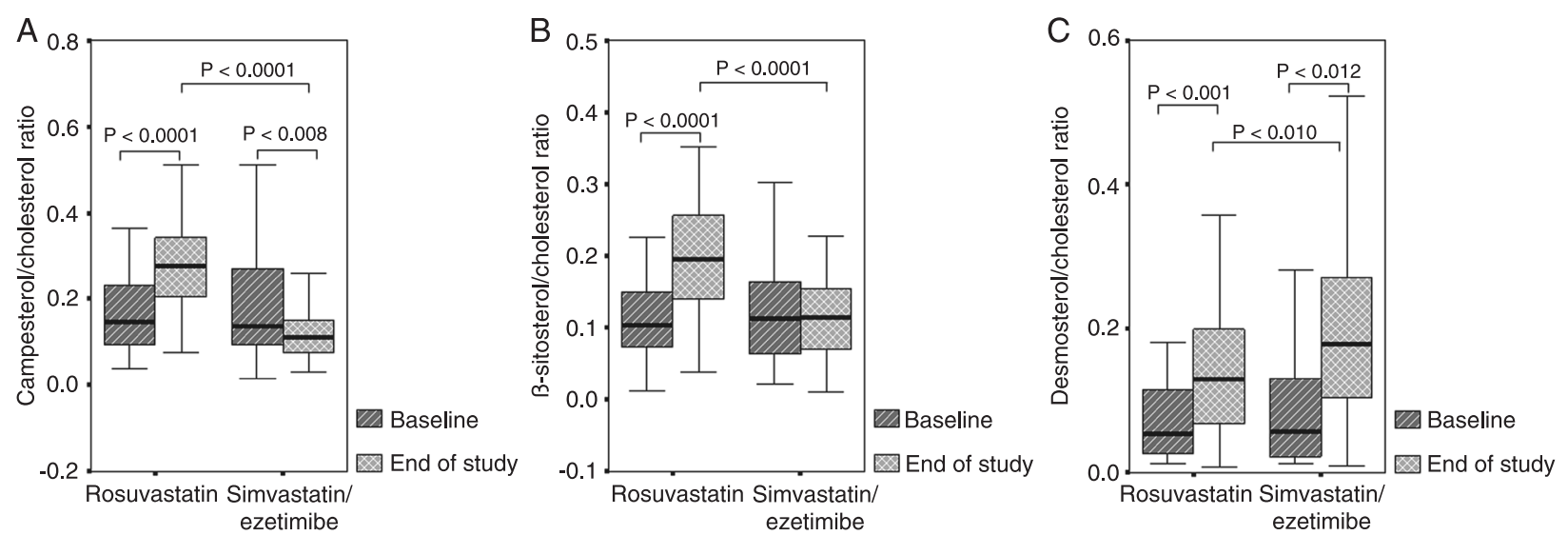

Figure 2. Box-plots (median, 25th and 75th percentiles) of the campesterol/cholesterol $(A)$, $\beta$-sitosterol/cholesterol $(B)$ and desmosterol/cholesterol $(C)$ ratios at baseline and at the end of the study according to treatment. Baseline values were comparable between groups. Rosuvastatin increased all of these ratios $(P<0.002$ vs baseline for all, Wilcoxon test). Treatment with simvastatin/ezetimibe promoted a significant decrease of the campesterol/cholesterol ratio $(P=0.008$ vs baseline, Wilcoxon test), but not of the $\beta$-sitosterol/ cholesterol ratio $(P=N S)$. Conversely, the desmosterol/cholesterol ratio increased 3-fold after treatment with simvastatin/ezetimibe $(P$ $<0.0001)$. Comparison of the effects of treatments showed that campesterol/cholesterol and $\beta$-sitosterol/cholesterol ratios were lower at the end of treatment in patients receiving simvastatin/ezetimibe than in those treated with rosuvastatin $(P<0.0001$, Mann-Whitney test), whereas the desmosterol/cholesterol ratio was higher in patients treated with simvastatin/ezetimibe $(P<0.0001)$.

plant sterols for incorporation into micelles, $\beta$-sitosterol was reported to reduce the expression of the Niemann-PickC1like 1 (NPC1L1) transporter (31). Therefore, in our study, a less pronounced increase in phytosterol levels may have been influenced by the adherence of these subjects to the diet in both arms.

Recently, a meta-analysis of data from 170,000 subjects in 26 randomized studies confirmed the benefit and safety of LDL cholesterol lowering using statins, and suggested that the reduction of 2-3 mM would reduce major cardiovascular events by about $40-50 \%$ (1). On the other hand, the benefits of LDL cholesterol lowering using other strategies seem less clear (32-34).

Our results showed that two highly effective lipid-lowering strategies induced a similar reduction of LDL cholesterol

\section{References}

1. Baigent C, Blackwell L, Emberson J, Holland LE, Reith C, Bhala N, et al. Efficacy and safety of more intensive lowering of LDL cholesterol: a meta-analysis of data from 170,000 participants in 26 randomised trials. Lancet 2010; 376: 16701681.

2. Jones $\mathrm{PH}$, Davidson $\mathrm{MH}$, Stein EA, Bays HE, McKenney $\mathrm{JM}$, Miller E, et al. Comparison of the efficacy and safety of rosuvastatin versus atorvastatin, simvastatin, and pravastatin across doses (STELLAR* Trial). Am J Cardiol 2003; 92: 152-160.

3. Ballantyne CM, Weiss R, Moccetti T, Vogt A, Eber B, Sosef $\mathrm{F}$, et al. Efficacy and safety of rosuvastatin $40 \mathrm{mg}$ alone or in combination with ezetimibe in patients at high risk of levels. However, substantial differences were found in the metabolism of sterols. Since differences in cholesterol homeostasis characterized by the balance between sterol absorption and synthesis are related to the severity of coronary disease, these differences may explain the unexpected findings in recent clinical trials. Further studies addressing cardiovascular events are needed to assess the impact of such differences on sterol metabolism.

\section{Acknowledgments}

Research supported by FAPESP (\#2008/55443-6) and CNPq (\#2008/57685-7). S.H. Kasmas was the recipient of a grant from FAPESP (\#2008/52597-2).

cardiovascular disease (results from the EXPLORER study). Am J Cardiol 2007; 99: 673-680.

4. Assmann G, Kannenberg F, Ramey DR, Musliner TA, Gutkin SW, Veltri EP. Effects of ezetimibe, simvastatin, atorvastatin, and ezetimibe-statin therapies on non-cholesterol sterols in patients with primary hypercholesterolemia. Curr Med Res Opin 2008; 24: 249-259.

5. van Himbergen TM, Matthan NR, Resteghini NA, Otokozawa $\mathrm{S}$, Ai M, Stein EA, et al. Comparison of the effects of maximal dose atorvastatin and rosuvastatin therapy on cholesterol synthesis and absorption markers. J Lipid Res 2009; 50: 730-739.

6. Jakulj L, Vissers MN, Groen AK, Hutten BA, Lutjohann D, 
Veltri EP, et al. Baseline cholesterol absorption and the response to ezetimibe/simvastatin therapy: a post-hoc analysis of the ENHANCE trial. J Lipid Res 2010; 51: 755-762.

7. Jakulj L, Vissers MN, van Roomen CP, van der Veen, Vrins $\mathrm{CL}$, Kunne $\mathrm{C}$, et al. Ezetimibe stimulates faecal neutral sterol excretion depending on abcg8 function in mice. FEBS Lett 2010; 584: 3625-3628.

8. Kosoglou T, Statkevich P, Johnson-Levonas AO, Paolini JF, Bergman AJ, Alton KB. Ezetimibe: a review of its metabolism, pharmacokinetics and drug interactions. Clin Pharmacokinet 2005; 44: 467-494.

9. Lennernas H, Fager G. Pharmacodynamics and pharmacokinetics of the HMG-CoA reductase inhibitors. Similarities and differences. Clin Pharmacokinet 1997; 32: 403-425.

10. Matthan NR, Resteghini N, Robertson M, Ford I, Shepherd $\mathrm{J}$, Packard C, et al. Cholesterol absorption and synthesis markers in individuals with and without a CHD event during pravastatin therapy: insights from the PROSPER trial. J Lipid Res 2010; 51: 202-209.

11. Wang CY, Liu PY, Liao JK. Pleiotropic effects of statin therapy: molecular mechanisms and clinical results. Trends Mol Med 2008; 14: 37-44.

12. Liu PY, Liu YW, Lin LJ, Chen JH, Liao JK. Evidence for statin pleiotropy in humans: differential effects of statins and ezetimibe on rho-associated coiled-coil containing protein kinase activity, endothelial function, and inflammation. Circulation 2009; 119: 131-138.

13. Zhou Q, Liao JK. Pleiotropic effects of statins. - Basic research and clinical perspectives. Circ J 2010; 74: 818-826.

14. Bellosta S, Paoletti R, Corsini A. Safety of statins: focus on clinical pharmacokinetics and drug interactions. Circulation 2004; 109: III-50-III-57.

15. Rubba P, Marotta G, Gentile M. Efficacy and safety of rosuvastatin in the management of dyslipidemia. Vasc Health Risk Manag 2009; 5: 343-352.

16. Third Report of the National Cholesterol Education Program (NCEP) Expert Panel on Detection, Evaluation, and Treatment of High Blood Cholesterol in Adults (Adult Treatment Panel III) final report. Circulation 2002; 106: 3143-3421.

17. Friedewald WT, Levy RI, Fredrickson DS. Estimation of the concentration of low-density lipoprotein cholesterol in plasma, without use of the preparative ultracentrifuge. Clin Chem 1972; 18: 499-502.

18. Ramos SC, Fonseca FA, Kasmas SH, Moreira FT, Helfenstein T, Borges NC, et al. The role of soluble fiber intake in patients under highly effective lipid-lowering therapy. Nutr $\mathrm{J}$ 2011; 10: 80.

19. Feio CA, Izar MC, Ihara SS, Kasmas SH, Martins CM, Feio $\mathrm{MN}$, et al. Euterpe oleracea (Acai) modifies sterol metabolism and attenuates experimentally-induced atherosclerosis. J Atheroscler Thromb 2012; 19: 237-245.

20. Kajinami K, Brousseau ME, Nartsupha C, Ordovas JM, Schaefer EJ. ATP binding cassette transporter G5 and G8 genotypes and plasma lipoprotein levels before and after treatment with atorvastatin. J Lipid Res 2004; 45: 653-656.

21. Guyton JR, Betteridge DJ, Farnier M, Leiter LA, Lin J, Shah
A, et al. Achievement of recommended lipid and lipoprotein levels with combined ezetimibe/statin therapy versus statin alone in patients with and without diabetes. Diab Vasc Dis Res 2011; 8: 160-172.

22. Silbernagel G, Fauler G, Renner W, Landl EM, Hoffmann MM, Winkelmann BR, et al. The relationships of cholesterol metabolism and plasma plant sterols with the severity of coronary artery disease. J Lipid Res 2009; 50: 334-341.

23. Matthan NR, Pencina M, LaRocque JM, Jacques PF, D'Agostino RB, Schaefer EJ, et al. Alterations in cholesterol absorption/synthesis markers characterize Framingham offspring study participants with CHD. J Lipid Res 2009; 50: 1927-1935.

24. Nunes VS, Leanca CC, Panzoldo NB, Parra E, Cazita PM, Nakandakare ER, et al. HDL-C concentration is related to markers of absorption and of cholesterol synthesis: Study in subjects with low vs. high HDL-C. Clin Chim Acta 2011; 412: 176-180.

25. Bhattacharyya AK, Connor WE. Beta-sitosterolemia and xanthomatosis. A newly described lipid storage disease in two sisters. J Clin Invest 1974; 53: 1033-1043.

26. Salen G, Horak I, Rothkopf M, Cohen JL, Speck J, Tint GS, et al. Lethal atherosclerosis associated with abnormal plasma and tissue sterol composition in sitosterolemia with xanthomatosis. J Lipid Res 1985; 26: 1126-1133.

27. Assmann G, Cullen P, Erbey J, Ramey DR, Kannenberg F, Schulte $\mathrm{H}$. Plasma sitosterol elevations are associated with an increased incidence of coronary events in men: results of a nested case-control analysis of the Prospective Cardiovascular Munster (PROCAM) study. Nutr Metab Cardiovasc Dis 2006; 16: 13-21.

28. Miettinen TA, Gylling $\mathrm{H}$. The effects of statins and sitosterols: benefit or not? Curr Atheroscler Rep 2009; 11: 23-27.

29. Strandberg TE, Gylling H, Tilvis RS, Miettinen TA. Serum plant and other noncholesterol sterols, cholesterol metabolism and 22-year mortality among middle-aged men. Atherosclerosis 2010; 210: 282-287.

30. Izar MC, Tegani DM, Kasmas SH, Fonseca FA. Phytosterols and phytosterolemia: gene-diet interactions. Genes Nutr 2011; 6: 17-26.

31. Jesch ED, Seo JM, Carr TP, Lee JY. Sitosterol reduces messenger RNA and protein expression levels of Niemann-Pick C1-like 1 in FHs 74 Int cells. Nutr Res 2009; 29: 859-866.

32. Rossebo AB, Pedersen TR, Boman K, Brudi P, Chambers $\mathrm{JB}$, Egstrup $\mathrm{K}$, et al. Intensive lipid lowering with simvastatin and ezetimibe in aortic stenosis. N Engl J Med 2008; 359: 1343-1356.

33. Kastelein JJ, Akdim F, Stroes ES, Zwinderman AH, Bots ML, Stalenhoef AF, et al. Simvastatin with or without ezetimibe in familial hypercholesterolemia. N Engl J Med 2008; 358: $1431-1443$

34. Taylor AJ, Villines TC, Stanek EJ, Devine PJ, Griffen L, Miller $\mathrm{M}$, et al. Extended-release niacin or ezetimibe and carotid intima-media thickness. N Engl J Med 2009; 361: 21132122 